\title{
Do electromagnetic effects survive in the production of lepton pairs in nucleus-nucleus collisions?
}

\author{
Antoni Szczurek* \\ Institute of Nuclear Physics PAN \\ E-mail: antoni.szczurek@ifj.edu.pl
}

We discuss production of $J / \psi$ and $e^{+} e^{-}$pairs in semicentral and peripheral collisions of heavy ions at high energies. We focus on photoproduction mechanism. We present explanation of results of the ALICE collaboration for low transverse momentum production of $J / \psi$ as well as results obtained by the STAR collaboration for $e^{+} e^{-}$production, also at low transverse momenta. We conclude that such effects are important also in this new corner (semi-central processes) of the phase space.

European Physical Society Conference on High Energy Physics - EPS-HEP2019 -

10-17 July, 2019

Ghent, Belgium

${ }^{*}$ Speaker. 


\section{Introduction}

Both vector meson and dilepton production are flag processes in ultraperipheral nucleusnucleus collisions. In these processes nuclei do not collide directly one with each other. Only particles of interest (vector mesons or dileptons) are produced. Such processes are characterised by small multiplicity. Those processes are induced by strong electromagnetic fields sourounding colliding high-energy heavy ions. Both vector meson production and dilepton production was studied in traditional nuclear collisions in a broad range of collisions energies. At high energy collisions one is interested in making a relation to production of quark-gluon plasma which is a traditional topic of interest in the field.

Three years ago the ALICE collaboration observed $J / \psi$ with very small transverse momenta in peripheral and semi-central collisions [1]. This was interpreted in [2] as effect of photoproduction mechanism which is active also in such a case.

Last year the STAR collaboration observed also enhanced production of dielectron pairs with small transverse momenta [3]. Last year we showed [4] that this may be interpreted as $\gamma \gamma \rightarrow e^{+} e^{-}$ processes (with coherent photons) even in the semi-central collisions.

In this presentation we briefly review the new processes, which we call semicentral to be distinguished from central or ultraperipheral collisions extensively discussed in the literature.

\section{A sketch of the formalism}

We start by showing the general situation in the impact parameter space.

In Fig. 1 we show the situation for $J / \psi$ production. The production of meson "happens" in the second nucleus. There are two contributions: (a) either the first (left) or (b) the second (right) nucleus emits a foton which fluctuates and rescatter in the partner nucleus. There is a region of overlapping nuclear densities where quark-gluon plasma is created. Can vector meson $(J / \psi$ in our case) be produced then? A comparison with experimental data may answer this question.
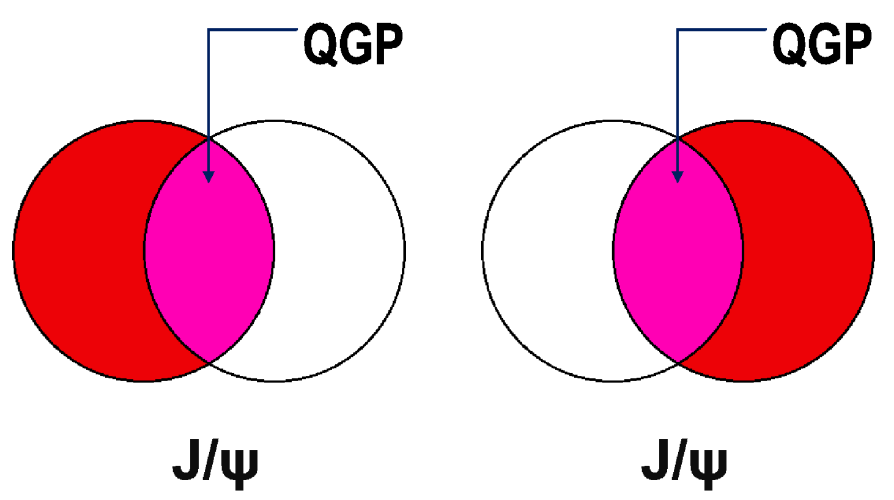

Figure 1: Emission of $J / \psi-$ a picture in the plane $x, y$ perpendicular to the collision axis $(z)$. In the gray area quark-gluon plasma is created.

A $b$-space picture for dileption production is represented in Fig.2. In general, the dilepton pair may be produced everywhere in the $\left(b_{x}, b_{y}\right)$ space. It is worth of noting that a big contribution may come from the red region, i.e. outside of the nuclei. Other regions have less sure status. 


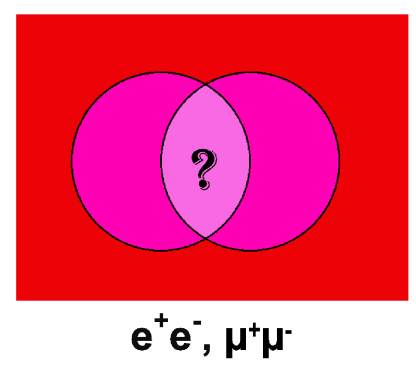

Figure 2: Dilepton production - a picture in the plane $x, y$ perpendicular to the collision axis $(z)$. The area with the question mark is the region where quark-gluon plasma is created.

The main ingredient for the photon-photon fusion mechanism is the flux of photons for an ion of charge $Z$ moving along $z$-axis at a given impact parameter with the relativistic parameter $\gamma$. With the nuclear charge form factor $F_{\mathrm{em}}$ as an input the flux can be calculated as $[5,6]$

$$
N(\omega, b)=\frac{Z^{2} \alpha_{\mathrm{EM}}}{\pi^{2}}\left|\int_{0}^{\infty} d q_{t} \frac{q_{t}^{2} F_{\mathrm{em}}\left(q_{t}^{2}+\frac{\omega^{2}}{\gamma^{2}}\right)}{q_{t}^{2}+\frac{\omega^{2}}{\gamma^{2}}} J_{1}\left(b q_{t}\right)\right|^{2},
$$

where $J_{1}$ is a Bessel function, $q_{t}$ is photon transverse momentum and $\omega$ is photon energy. We calculate the form factor from the Fourier transform of the nuclear charge density.

The differential cross section for dilepton $\left(l^{+} l^{-}\right)$production via $\gamma \gamma$ fusion at fixed impact parameter of a nucleus nucleus collision can then be written as

$$
\frac{d \sigma_{l l}}{d \xi d^{2} b}=\int d^{2} b_{1} d^{2} b_{2} \delta^{(2)}\left(\vec{b}-\vec{b}_{1}-\vec{b}_{2}\right) N\left(\omega_{1}, b_{1}\right) N\left(\omega_{2}, b_{2}\right) \frac{d \sigma\left(\gamma \gamma \rightarrow l^{+} l^{-} ; \hat{s}\right)}{d p_{t}^{2}}
$$

where the phase space element is $d \xi=d y_{+} d y_{-} d p_{t}^{2}$ with $y_{ \pm}, p_{t}$ and $m_{l}$ the single-lepton rapidities, transverse momentum and mass, respectively, and

$$
\omega_{1}=\frac{\sqrt{p_{t}^{2}+m_{l}^{2}}}{2}\left(e^{y_{+}}+e^{y_{-}}\right), \omega_{2}=\frac{\sqrt{p_{t}^{2}+m_{l}^{2}}}{2}\left(e^{-y_{+}}+e^{-y_{-}}\right), \hat{s}=4 \omega_{1} \omega_{2} .
$$

As can be seen from Eq.(2.1), the transverse momenta of the photons have been integrated out, and in this approximation dileptons are produced back-to-back in the transverse plane.

An exact calculation of the pair- $P_{T}$ dependence is, in general, rather involved. In Ref.[4] we performed a simplified calculation using $b$-integrated transverse momentum dependent photon fluxes.

\section{Selected results}

\section{$3.1 J / \psi$ production}

In Fig. 3 we show our results (cross section in the ALICE rapidity interval) starting from centralities bigger than $30 \%$. The ALICE Collaboration could not extract actual values of the cross section for the two lowest centrality bins. The results when using standard photon fluxes exceed 


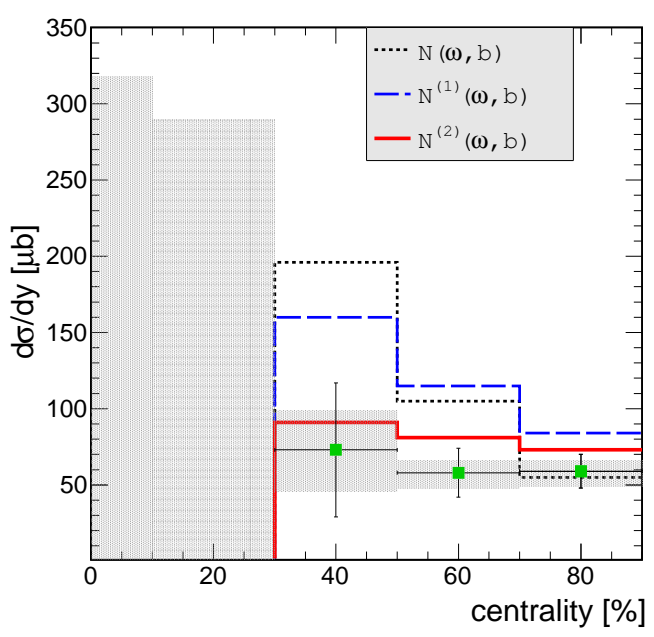

Figure 3: $\Delta \sigma / \Delta y$ cross sections for different centrality bins. Theoretical results for different models of the photon flux are compared with the ALICE data [1]. The shaded area represents the experimental uncertainties.

the ALICE data. Rather good agreement with the data is achieved when the overlap region in Fig.1 is removed (which corresponds to $N^{(2)}$ in [2]).

The $J / \psi$ corresponding to the photoproduction mechanism are concentrated in the region of very small transverse momenta. So far, according to my knowledge, there were no attempts to describe transverse momentum distributions of $J / \psi$ mesons. In the $b$-space approach it is just the Dirac delta.

\section{2 $e^{+} e^{-}$production}

The dielectron invariant-mass distribution is shown in Fig.4. Here we show results for $P_{T}<$ $0.15 \mathrm{GeV}$ (transverse momentum of the pair) and three different centrality classes as selected in the STAR analysis: peripheral (60-80\%), semi-peripheral (40-60\%) and semi-central (10-40\%) collisions. In peripheral collisions the photon-photon contribution dominates while in semi-central collisions all three contributions are of similar magnitude. Their sum is in good agreement with the STAR data, except for the $J / \psi$ peak region. Our calculations contain only incoherent $J / \psi$ production, from binary nucleon-nucleon collisions. We conjecture that the missing contribution is due to a coherent contribution [2].

Can we describe also transverse momentum distributions of the $e^{+} e^{-}$pair ? The $b$-space approach sketched above is not enough to calculate transverse momentum distributions of the pair which is a Dirac delta function in the approximation made there. To calculate the distributions in transverse momentum of the pair one needs rather momentum approach. In our recent paper we considered a $k_{t}$-factorization approach. In this approach one considers fluxes of photons which depend on photon energies and photon transverse momenta. In this way one gets rather shapes of the transverse momentum distributions. The normalization in a given bin of centrality may be taken from the $b$-space calculation. Our results are shown in Fig.5. We show also contributions of other processes considered in [4]. 

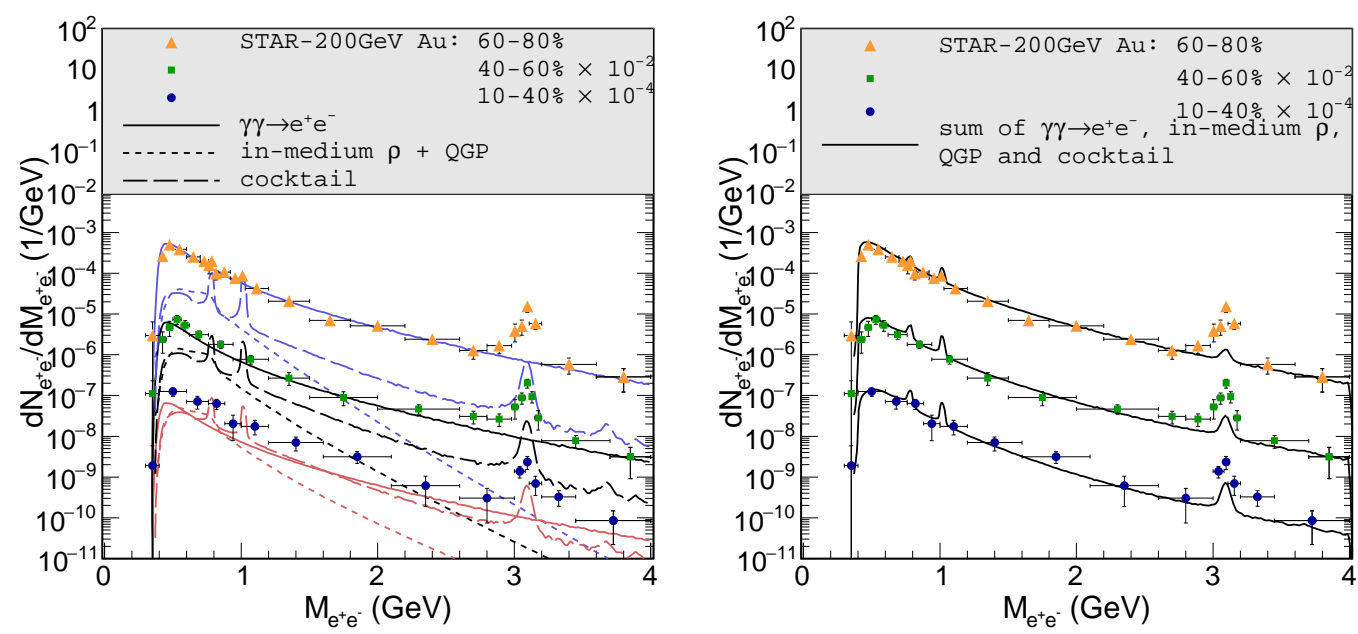

Figure 4: Left: Dielectron invariant-mass spectra for pair- $P_{T}<0.15 \mathrm{GeV}$ in $\mathrm{Au}+\mathrm{Au}\left(\sqrt{s_{N N}}=200 \mathrm{GeV}\right)$ collisions for 3 centrality classes including experimental acceptance cuts $\left(p_{t}>0.2 \mathrm{GeV},\left|\eta_{e}\right|<1\right.$ and $\left.\left|y_{e^{+}} e^{-}\right|<1\right)$ for $\gamma \gamma$ fusion (solid lines), thermal radiation (dotted lines) and the hadronic cocktail (dashed lines); right panel: comparison of the total sum (solid lines) to the STAR data [3].

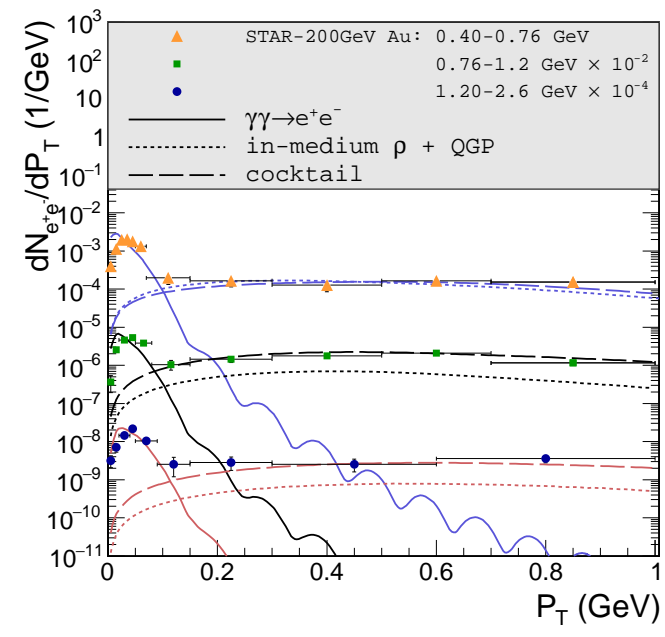

Figure 5: $P_{T}$ spectra of the individual contributions (line styles as in the previous figure) in 3 different mass bins for the $60-80 \%$ centrality of the Au+Au collisions $\left(\sqrt{s_{N N}}=200 \mathrm{GeV}\right)$, compared to the STAR data [3].

In general, there could be also dependence of transverse momentum distribution of the pair on impact parameter (centrality of the collision). In our opinion there is no correct formalism in the literature in this respect. We will discuss this issue elsewhere.

\section{Conclusions}

Here I have briefly reviewed a new category of processes discovered in last three years. I have started my presentation from low transverse momentum production of $J / \psi$. It was shown that cross 
section for such processes strongly depends on the region of impact parameter space. In particular, the region (in impact parameter space) of overlapping nuclear densities is interesting as there quarkgluon plasma is created. We have discussed a potential effect of the plasma on production of $J / \psi$. By comparing to the ALICE data we are inclined to conclude that the production of $J / \psi$ in this region seems to be suppressed. This provides a new information on the space-time picture of the vector meson creation.

I have presented also results for dielectron production for RHIC energies. The low transverse momentum dielectron production can be understood as due to $\gamma \gamma \rightarrow e^{+} e^{-}$subrocess in heavy ion collisions. We obtained a good agreement with the STAR data for different centralities. I have pointed out the essential geometrical difference between $e^{+} e^{-}$and $J / \psi$ production. In our recent paper we presented also first trial to understand transverse momentum distributions of $e^{+} e^{-}$pairs. This is a very interesting observable which requires further attention in the future.

\section{Acknowledgement}

I am indebted to Mariola Kłusek-Gawenda, Ralf Rapp and Wolfgang Schäfer for collaboration on the issues presented here.

\section{References}

[1] E.L. Kryshen for the collaboration [ALICE Collaboration], Nucl. Phys. A967 (2017) 273.

[2] M. Kłusek-Gawenda and A. Szczurek, "Photoproduction of $J / \psi$ mesons in peripheral and semicentral heavy ion collisions", Phys. Rev. C93 (2016) 044912.

[3] J. Adam et al. [STAR Collaboration], Phys. Rev. Lett. 121 (2018) 132301.

[4] M. Kłusek-Gawenda, R. Rapp, W. Schäfer and A. Szczurek, "Dilepton Radiation in Heavy-Ion Collisions at Small Transverse Momentum", Phys. Lett. B790 (2019) 339.

[5] C. A. Bertulani and G. Baur, Phys. Rept. 163 (1988) 299.

[6] G. Baur, K. Hencken, D. Trautmann, S. Sadovsky and Y. Kharlov, Phys. Rept. 364 (2002) 359. 